\title{
A 7-Year Trend of Malaria at Primary Health Facilities in Northwest Ethiopia
}

\author{
Ayenew Addisu (D), Yalewayker Tegegne $\mathbb{D}^{D}$, Yenesew Mihiret, Abebaw Setegn, \\ and Ayalew Jejaw Zeleke
}

\begin{abstract}
Department of Medical Parasitology, School of Biomedical and Laboratory Sciences, College of Medicine and Health Sciences, University of Gondar, Gondar, Ethiopia
\end{abstract}

Correspondence should be addressed to Ayenew Addisu; ayenew.addisu@gmail.com

Received 24 July 2019; Revised 2 November 2019; Accepted 3 December 2019; Published 29 January 2020

Academic Editor: José F. Silveira

Copyright ( 2020 Ayenew Addisu et al. This is an open access article distributed under the Creative Commons Attribution License, which permits unrestricted use, distribution, and reproduction in any medium, provided the original work is properly cited.

\begin{abstract}
Background. Malaria is a severe parasitic disease that can progress to complications of the nervous system, respiratory distress, renal problems, metabolic acidosis, and hypoglycemia which can result in death in case of delay or absence of appropriate treatment. Even though health service facilities and vector control strategy in the community are implemented as control measures, variations in temperature and rainfall that can affect the life cycle of parasite are among the factors of malaria prevalence over the years. The aim of this trend analysis was to assess the prevalence and the impact of malaria over the seasons and years. Methods. A cross-sectional study using retrospective information was conducted at two health centers Gorgora and Chuahit in Dembia district. The data was collected from lab logbooks routinely diagnosed and registered for seven years. A systematic sampling technique was used by taking patient results from lab logbooks during the first ten days of every month. Data were entered directly into the EpiData Entry software version 3.1 and analysed with the SPSS software version 20. Moreover, a chi-square test with a level of significance set at less than $5 \%$ was used. Results. From a total of 11,879 clients that participated, $56.6 \%$ were males. The overall malaria prevalence in the last seven years was $21.8 \%$, and the dominant parasite was $P$. falciparum which accounted for $15.6 \%$ of the participants which was threefold higher than $P$. vivax in the seven-year trend. Moreover, at Gorgora health center, the prevalence which was 15\% at the beginning of the study (2012) rose to $33.9 \%$ and $30.5 \%$ in 2017 and 2018 , respectively. In the analysis of the seven years, October and September in which the prevalence of malaria was $32.6 \%$ and $27.2 \%$, respectively, constituted the peak months. High malaria prevalence was observed in autumn (September to November) season, and the least was observed in spring (March to May) with the prevalence of (17.8\%) $(p \leq 0.001)$. Malaria attack showed significant variability among different age groups, and the age group 15-29 and males were the most affected $(p \leq 0.001)$. Conclusion. In this study, malaria transmission remained high, which affected males more than females. Thus, appropriate season-based bed net use, health education, immediate patient treatment, and stagnant water drainage methods are needed to alleviate the problem.
\end{abstract}

\section{Background}

Malaria is a life-threatening protozoan disease caused by plasmodium parasites that transmit to people through the bites of infected female Anopheles mosquitoes. Five Plasmodium parasitic species cause malaria in humans; of these, two species, Plasmodium falciparum and Plasmodium vivax, are predominant [1-3].

Malaria is a severe disease, which even progresses to complications and death if there is a delay or absence of appropriate treatment. Some of the clinical complications and manifestations observed in malaria infection are nervous system involvement, respiratory distress, renal problems, metabolic acidosis, and hypoglycemia [4].

Malaria infection affects nearly half of the world's population. As per the 2018 World malaria report, there were 219 million malaria cases, of which the 10 highest victims were African countries. It affects the lives of more than 435,000 people, the majority of whom are in Africa each year. Pregnant women and children under five years are among the 
most vulnerable groups [5]. To reduce malaria mortality in 15 high-burden sub-Saharan countries by $50 \%$, it requires an artemisinin-based combination therapy to create malariafree zones in Africa by 2040-2050 [6].

In Ethiopia, the overall trends of malaria cases and deaths among under-five children decreased by $59.3 \%$ and $72.1 \%$, respectively. On the other hand, malaria incidence rate decreased from an average of 43.1 to 29 cases and 2.1 to 1.1 deaths per 1000 people annually between 2001 and 2016 [7]. According to the 2016 Ethiopian malaria intervention report, there were $64 \%$ and $92.5 \%$ coverage of indoor residual spray (IRS) and insecticide-treated net (ITN), respectively [7].

Malaria transmission control strategies are very complex issues, influenced by various factors that may relate to the host, the parasite, the environment, the vector, and the health system capacity to fully implement the available rapid diagnostic tests and professional skill in microscopic species detection [5]. Besides, the most effective prevention and control strategies are active case management, operational research and surveillance, and monitoring and evaluation systems to provide appropriate information [5].

Therefore, the prevalence of malaria is expected to shift over the years because of the increment in the number of health service facilities, high vector control strategy coverage by using indoor residual spray and insecticide-treated nets in the community, and the awareness of the rural population about health seeking behavior [8]. Besides this, to accelerate malaria control strategy, considering seasonal malaria prevalence variability over the years is crucial.

Information from specific health facility laboratory registers over the long periods of time is valuable for a better malaria control strategy. Therefore, this long-term malaria parasite trend over the years and the variability within a year in laboratories of two primary health facilities have provided valuable information for further action.

\section{Methods}

2.1. Study Area, Population, and Period. Dembia district is located in the Amhara region of Ethiopia $35 \mathrm{~km}$ from the Gondar town. It was bordered by Gondar town and Takusa, Chilga, and Belesa districts. Dembia has a total population of 307,967 in an area of $148,968 \mathrm{sq}$. $\mathrm{km}$. Its altitude ranges from 1,850 to $2,000 \mathrm{~m}$ above sea level with an average annual rainfall of 700 to $1,160 \mathrm{~mm}$. It has 10 health centers and 40 health posts. Among the ten health centers, Gorgora and Chuahit were chosen for this study. Each health center was $16 \mathrm{~km}$ apart, and Gorgora was bordered by Lake Tana. The study participants were all clients who provided blood samples for laboratory blood film microscopic examinations and registered on the lab logbooks of the two health centers $[9,10]$.

\subsection{Study Design, Sampling Technique, and Data Collection.} A cross-sectional study using retrospective information was carried out in the two health centers of Dembia district. The data was collected from seven years of registered lab logbook that stained and diagnosed with Giemsa's staining technique. Since taking the whole examined blood films over seven years in the two health centers is cumbersome, we systematically took patient results of the first ten days of every month.

2.3. Analysis and Statistics. Data from the laboratory registered logbook was entered directly into the EpiData Entry software version 3.1 and analysed with the SPSS software version 20. Excel was also used to summarize some figures. Descriptive statistics were used to summarize the data and to display malaria parasite trends over the years and seasonal variations. We compared parasite proportions by gender, age groups, seasons of the year, and trends over the years. Moreover, a chi-square test with a level of significance set at less than $5 \%$ was considered.

\section{Results}

A total of 11,879 clients were requested for blood film and registered on lab logbooks of the two health centers. Of whom, 6, 724 (56.6) were males and 7, 859 (66.2\%) were enrolled from Chuahit health center. The total malaria prevalence over the seven years was $21.8 \%$, of which $P$. falciparum that accounted for $15.6 \%$ was the dominant parasitic species, followed by 733 (5.3\%) of $P$. vivax. The rest 101 (0.9\%) were mixed or both $P$. falciparum and $P$. vivax infections.

3.1. Malaria Trend in Different Years and Seasons. Over the last seven years, the prevalence of malaria was highly variable across years ranging from $14 \%$ to $30.2 \%(p \leq 0.001)$. The highest prevalence was observed in 2016 (30.2\%) followed by $2015(24 \%)$. Of the twelve months of the seven years, October had the highest prevalence (32.6\%) followed by September (27.2\%). On the other hand, the least prevalence was observed in February (15.1\%). As far as malaria prevalence across the different seasons was concerned, the highest prevalence was observed during autumn (September to November) (27.9\%), followed by 23.3 and $18.4 \%$ in summer (June to August) and winter (December to February), respectively. Spring (March to May) was the season with the least prevalence $(17.8 \%)(p \leq 0.001)$ (Figure 1).

3.2. Malaria Distribution in relation to Age and Sex. The prevalence of malaria varied among different age groups ranging from $16.3 \%$ to $25.5 \%(p \leq 0.001)$. The $15-29$-year age group was the most affected followed by the 5-14-year group with the prevalence of $25.5 \%$ and $24.6 \%$, respectively. The least malaria prevalence was observed among 50-59 years of the age group (16.3\%). In this study, males were more affected than females over the seven years, which ranged from $17.4 \%$ to $33.7 \%$ as shown in Figures 2 (a) and 2(b).

3.3. Plasmodium Species Distribution among People and Health Centers. P. falciparum was threefold more dominant than $P$. vivax in the seven-year trend $(15.6 \%$ and $5.3 \%$, respectively). Moreover, at Gorgora health center, the prevalence which was $15 \%$ at the beginning of the study (2012) rose to $33.9 \%$ and $30.5 \%$ in 2017 and 2018, respectively. On the other hand, at Chuahit health center, the prevalence decreased from $27.4 \%$ in 2012 to $11.6 \%$ at the end of 2018 , although after high prevalence was seen in 2016 (Figure 3). 


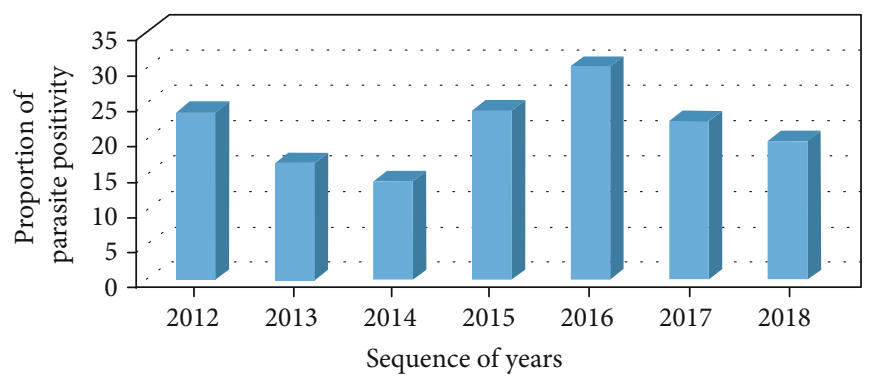

(a)

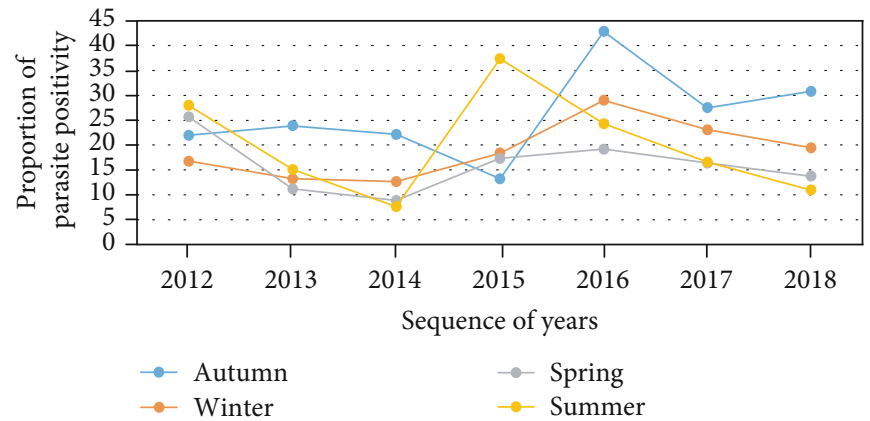

(b)

FigURE 1: Seasonal variations and trend prevalence of malaria infection over the study period in Dembia district health centers from 2012 to 2018. (a) Annual trend prevalence. (b) Positivity rate across seasons $(n=11,879)$.

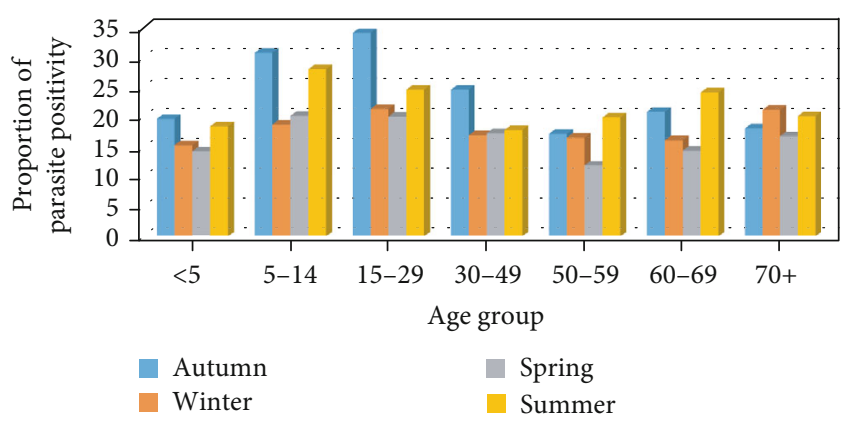

(a)

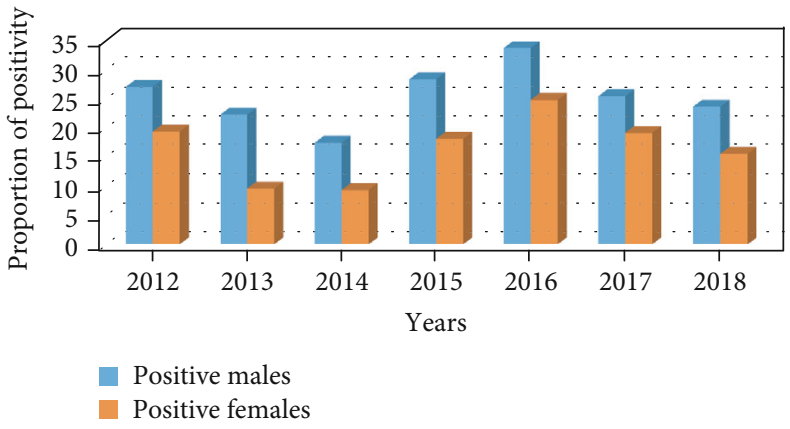

(b)

FIgURe 2: Malaria infection in different seasons with age groups and sex in Dembia district health centers from 2012 to 2018. (a) Malaria prevalence in different seasons with age groups. (b) Sex and malaria prevalence distribution $(n=11,879)$.

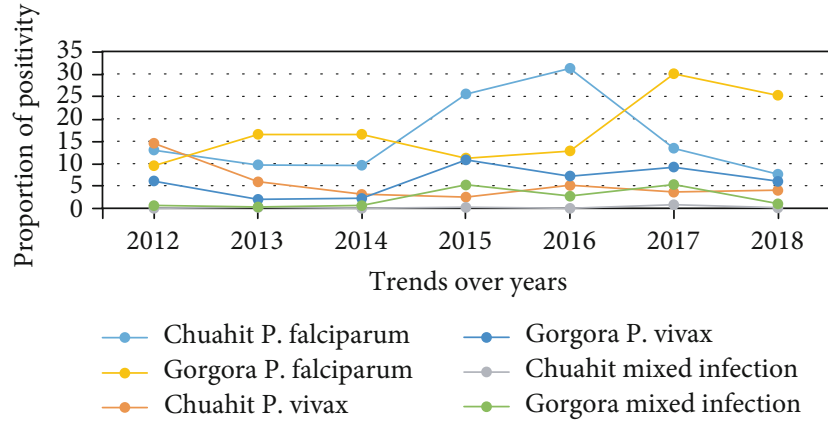

FIGURE 3: Plasmodium species distribution on the population and health centers in the last seven years in Dembia district health centers from 2012 to 2018.
In the study district, long-lasting insecticidal nets (LLIN) were distributed in proportion to family members every three years. Carbamate group chemicals bendiocarb and propoxur wettable powders were used to control mosquito vectors over the last seven years. Either one of the two or combined composition of bendiocarb and propoxur chemicals was for indoor residual spray to control mosquito landing. Table 1 shows the houses sprayed for seven years and population expected to have been protected.

\section{Discussion}

The purpose of this trend analysis was to investigate the spread and impact of malaria across the nations of Ethiopia and seasons from 2012 to 2018 in order to highlight priorities 
TABLE 1: Insecticide distribution, number of sprayed houses, and number of protected population in Dembia district from 2012 to 2018.

\begin{tabular}{|c|c|c|c|c|c|}
\hline Study years & $\begin{array}{c}\text { No. of sprayed } \\
\text { houses }\end{array}$ & $\begin{array}{l}\text { Population protected } \\
\text { against mosquitoes }\end{array}$ & $\begin{array}{c}\text { Total } \\
\text { population }\end{array}$ & $\begin{array}{c}\text { No. of subcities covered } \\
\text { by chemical spray }\end{array}$ & Types of chemicals used \\
\hline 2012 & 45,351 & 250,530 & 295,802 & 47 & $\begin{array}{l}\text { Propoxur and } \\
\text { bendiocarb }\end{array}$ \\
\hline 2013 & 76,033 & 259,987 & 301,073 & 42 & Bendiocarb \\
\hline 2014 & 46,494 & 179,566 & 309,458 & 29 & Bendiocarb \\
\hline 2015 & 22,623 & 84,706 & 316,913 & 15 & Bendiocarb \\
\hline 2016 & 52,397 & 181,704 & 321,877 & 29 & Propoxur \\
\hline 2017 & 13,911 & 47,077 & 328,757 & 7 & Propoxur \\
\hline 2018 & 87,665 & 163,899 & 335,637 & 21 & Bendiocarb \\
\hline
\end{tabular}

for the Ethiopian Vector-borne Disease Control Program intended to free the nation from the disease.

According to this study, the trend of the prevalence of malaria among the 11,879 clients who were registered in the lab logbooks of the two health centers and requested for blood films over the seven years was $21.8 \%$. This result was markedly lower than $36.1 \%, 48 \%$, and $33.8 \%$ reported from the nearby localities of Addi Arkay health center, northwest Ethiopia; Woreta town, northwest Ethiopia; and Abeshge, south-central Ethiopia, respectively $[8,11,12]$. On the other hand, it was higher than the five-year trend study conducted in Ataye, North Shoa, Ethiopia; reported a total prevalence of $8.4 \%$, the seven-year retrospective malaria report from Metema hospital, northwest Ethiopia; detected a prevalence of $17 \%$, the fifteen-year study in Ethiopia; and noted a prevalence of $12.5 \%[13,14]$. The differences might be due to time variations of the studies, difference in insecticide application in the areas, variations in geographical locations, differences in population awareness about malaria bed net application, its transmission, and health seeking behavior.

During the study, the highest prevalence $30.2 \%$ was observed in 2016, followed by 2015 (24\%). These results were similar to the results of a study on malaria epidemiology and interventions in Ethiopia which reported a decrease in malaria incidence and death rate in Ethiopia from 2001 to 2016. But malaria case number and incidence reported in 2016 exceeded the WHO standard for "pre-elimination strategy" [7]. This might be due to the use of propoxur instead of bendiocarb as indoor residual spray insecticide or relatively decreased number of subcities covered by chemical spray (Table 1).

In the current study, the prevalence of malaria was high in October and the least in February over the seven years of twelve months. Seasonal fluctuations and high prevalence of malaria were observed in autumn (September to December) and the least prevalence in spring (March to May). This was in line with the findings of studies conducted in Ataye, North Shoa, Ethiopia (by Ethiopian Federal Ministry of Health); Addi Arkay health center (Ethiopia); Abeshge (south-central Ethiopia); and Metema hospital, northwest Ethiopia $[8,11,14]$. Such high malaria prevalence in autumn (September to December) might be related to the formation of stagnant water after the heavy rain season, favorable temperature, and high vegetation density for mosquito breeding.
On the other hand, the least prevalence was observed in spring (March to May); this might be due to drought.

The study also showed that the prevalence of malaria was high among males and 15-29 years of age groups over the seven years trend. This was similar to the findings of studies conducted in Addi Arkay health center; Ataye district, North Shoa; and Abeshge, south-central Ethiopia [8, 11, 14]. This might be due to the fact that productive age groups often engaged in farm activities in the fields. Males usually sleep outdoors to look after farms. In most Ethiopian communities' culture, females are restricted to home activities, like taking care of children and usually spend their time indoors.

Moreover, P. falciparum was more dominant parasite than $P$. vivax which it exceeded by over threefold. The finding was comparable with the results of studies in Addi Arkay, northwest Ethiopia; Ataye, North Shoa, Ethiopia (by Ethiopian Federal Ministry of Health); Abeshge, southcentral Ethiopia; Woreta town, northwest Ethiopia; and Metema hospital, northwest Ethiopia, which reported that $P$. falciparum was the dominant parasite over the study periods of each study [11-15]. The reason may be that the $P$. falciparum parasite can multiply rapidly by involving more than one parasite in a single red blood cell, colonizing all ages of the red blood cells without any selection, parasite-infected red blood cells (RBCs) can accumulate in various organs, and the availability of $P$. falciparum infected cases in communities $[16,17]$.

Malaria was more widespread in Gorgora health center compared to Chuahit over the seven years; the prevalence at the beginning of the study was $15 \%$ and the peak was $33.9 \%$ in 2017. At Chuahit health center, the prevalence declined from $27.4 \%$ in 2012 to $11.6 \%$ in 2018 . This might be due to geographical location as Gorgora is located nearer Lake Tana; the vegetation in the shore may be appropriate for mosquito breeding.

In this study area, long-lasting insecticidal nets (LLIN) and IRS were used, though the coverage of IRS was low. This result was similar to the result of a study conducted by the Malaria Epidemiology and Interventions in Ethiopia, and it was reported that IRS coverage was low nationwide with an average coverage of $23 \%$ between 2014 and 2016 [7].

Data collection in the two health centers on the whole examined blood films over seven years was cumbersome; we systematically took patient results of the first ten days of every 
month. Moreover, the collected data relayed on the laboratory logbook which lacks participants' body temperature and clinical presentations were the main limitations of the study.

\section{Conclusion}

In this study, malaria transmission remains high, especially in autumn and summer seasons. The most affected were the productive age groups with a high infection rate in males. The dominant parasite was $P$. falciparum; hence, appropriate vector control methods that target outdoor and indoor transmissions are needed, especially for males during high malaria transmission seasons to avoid factors that affect success of malaria elimination.

\section{Abbreviations}

IRS: Indoor residual spray

ITN: Insecticide-treated net.

\section{Data Availability}

All data generated or analysed during this study were included in this published article.

\section{Ethical Approval}

Ethical approval was obtained from the Ethics Committee of the University of Gondar, Ethiopia.

\section{Consent}

After having a discussion about the purpose and method of the study, verbal consent was sought from the Head of the district health office before the data collection.

\section{Conflicts of Interest}

The authors declare that they have no competing interests.

\section{Authors' Contributions}

AA conceived the study and involved in the data collection. YTA, YMW, and ASY involved in the data collection and analysis. AJZ involved in the data analysis and critically reviewed the manuscript. All authors reviewed and approved the manuscript.

\section{Acknowledgments}

We would like to thank Dembia district health office and Gorgora and Chuahit health centers for allowing us to do this research. We are also grateful to all data collectors.

\section{References}

[1] World Health Organization, Investing to overcome the global impact of neglected tropical diseases: third WHO report on neglected tropical diseases 2015, World Health Organization, 2015.
[2] A. Tajebe, G. Magoma, M. Aemero, and F. Kimani, "Detection of mixed infection level of Plasmodium falciparum and Plasmodium vivax by SYBR Green I-based real-time PCR in North Gondar, north-west Ethiopia," Malaria Journal, vol. 13, no. 1, p. $411,2014$.

[3] WHO, "Malaria," October 2019, https://www.who.int/ith/ diseases/malaria/en/.

[4] A. Trampuz, M. Jereb, I. Muzlovic, and R. M. Prabhu, "Clinical review: severe malaria," Critical Care, vol. 7, no. 4 , p. $315,2003$.

[5] WHO, Malaria report, WHO, 2018.

[6] R. M. Oxborough, "Trends in US President's malaria initiative-funded indoor residual spray coverage and insecticide choice in sub-Saharan Africa (2008-2015): urgent need for affordable, long-lasting insecticides," Malaria Journal, vol. 15, no. 1, article 146, 2016.

[7] H. S. Taffese, E. Hemming-Schroeder, C. Koepfli et al., "Malaria epidemiology and interventions in Ethiopia from 2001 to 2016," Infectious diseases of poverty, vol. 7, no. 1, p. 103, 2018.

[8] F. Yimer, A. Animut, B. Erko, and H. Mamo, "Past five-year trend, current prevalence and household knowledge, attitude and practice of malaria in Abeshge, south-central Ethiopia," Malaria Journal, vol. 14, no. 1, article 230, 2015.

[9] F. Agegnehu, A. Shimeka, F. Berihun, and M. Tamir, "Determinants of malaria infection in Dembia district, Northwest Ethiopia: a case-control study," BMC Public Health, vol. 18, no. 1, p. 480, 2018.

[10] H. A. Wale, T. Bekele, and G. Dalle, "Floristic diversity, regeneration status, and vegetation structure of woodlands in Metema area, Amhara National Regional State, Northwestern Ethiopia," Journal of Forestry Research, vol. 23, no. 3, pp. 391-398, 2012.

[11] H. Tesfa, A. G. Bayih, and A. J. Zeleke, "A 17-year trend analysis of malaria at Adi Arkay, north Gondar zone, Northwest Ethiopia," Malaria Journal, vol. 17, no. 1, article 155, 2018.

[12] A. Alelign, Z. Tekeste, and B. Petros, "Prevalence of malaria in Woreta town, Amhara region, Northwest Ethiopia over eight years," BMC Public Health, vol. 18, no. 1, p. 990, 2018.

[13] G. Ferede, A. Worku, A. Getaneh et al., "Prevalence of malaria from blood smears examination: a seven-year retrospective study from Metema hospital, Northwest Ethiopia," Malaria Research and Treatment, vol. 2013, Article ID 704730, 5 pages, 2013.

[14] D. G. Feleke, D. Gebretsadik, and A. Gebreweld, “Analysis of the trend of malaria prevalence in Ataye, North Shoa, Ethiopia between 2013 and 2017," Malaria Journal, vol. 17, no. 1, p. 323, 2018.

[15] "President's malaria initiative: ethiopia - malaria operational plan FY," 2019.

[16] J. Beeson and G. Brown, "Pathogenesis of Plasmodium falciparum malaria: the roles of parasite adhesion and antigenic variation," Cellular and Molecular Life Sciences CMLS, vol. 59, no. 2, pp. 258-271, 2002.

[17] A. S. Paul, E. S. Egan, and M. T. Duraisingh, "Host-parasite interactions that guide red blood cell invasion by malaria parasites," Current Opinion in Hematology, vol. 22, no. 3, pp. 220-226, 2015. 